\title{
Effects of rotation on the helium burning shell source in accreting white dwarfs
}

\author{
S.-C. Yoon ${ }^{1}$, N. Langer ${ }^{1}$, and S. Scheithauer ${ }^{2,3}$ \\ 1 Astronomical Institute, Utrecht University, Princetonplein 5, 3584 CC, Utrecht, The Netherlands \\ e-mail: S.C.Yoon@astro.uu.nl \\ 2 Institut für Physik, Universität Potsdam, 14415 Potsdam, Germany \\ 3 Present address: ZARM, University of Bremen Am Fallturm, 28359 Bremen, Germany
}

Received 25 February 2004 / Accepted 3 June 2004

\begin{abstract}
We investigate the effects of rotation on the behavior of the helium-burning shell source in accreting carbon-oxygen white dwarfs, in the context of the single degenerate Chandrasekhar mass progenitor scenario for type Ia supernovae (SNe Ia). We model the evolution of helium-accreting white dwarfs of initially $1 M_{\odot}$, assuming four different constant accretion rates $\left(2,3,5\right.$ and $\left.10 \times 10^{-7} M_{\odot} / y r\right)$. In a one-dimensional approximation, we compute the mass accretion and subsequent nuclear fusion of helium into carbon and oxygen, as well as angular momentum accretion, angular momentum transport inside the white dwarf, and rotationally induced chemical mixing. Our models show two major effects of rotation: a) The helium-burning nuclear shell source in the rotating models is much more stable than in corresponding non-rotating models - which increases the likelihood that accreting white dwarfs reach the stage of central carbon ignition. This effect is mainly due to rotationally induced mixing at the $\mathrm{CO} / \mathrm{He}$ interface which widens the shell source, and due to the centrifugal force lowering the density and degeneracy at the shell source location. b) The C/O-ratio in the layers which experience helium shell burning - which may affect the energy of an SN Ia explosion - is strongly decreased by the rotationally induced mixing of $\alpha$-particles into the carbon-rich layers. We discuss implications of our results for the evolution of SNe Ia progenitors.
\end{abstract}

Key words. nuclear reactions, nucleosynthesis, abundances - stars: evolution - stars: white dwarf - stars: rotation supernovae: individual: type Ia

\section{Introduction}

Accreting white dwarfs in close binary systems are considered to be responsible for such important astrophysical phenomena as nova explosions, cataclysmic variables and super-soft X-ray sources. Especially, there is general agreement that accreting $\mathrm{CO}$ white dwarfs are progenitors of type Ia supernovae (SNe Ia) (e.g., Woosley \& Weaver 1986; Branch et al. 1995; Wheeler 1996; Nomoto et al. 1997, Hillebrandt \& Niemeyer 2000; Livio 2001).

The fairly homogeneous properties of light curves and spectra of SNe Ia have made them a valuable distance indicator for determining cosmological parameters (e.g., Hamuy et al. 1996; Branch 1998; Leibundgut 2001). One of the most intriguing results obtained from $\mathrm{SNe}$ Ia data may be the recent suggestion of a finite cosmological constant, which implies that the universe is currently accelerating its expansion (Perlmutter et al. 1999; Riess et al. 2000).

The observational properties of SNe Ia demand a thermonuclear explosion of a $\mathrm{CO}$ white dwarf as their origin. However, despite the far-reaching observational achievements, self-consistent progenitor evolution model calculations which explain the existence of $\mathrm{SNe}$ Ia are still lacking (but see
Yoon \& Langer 2003). Models for the evolution of SN Ia progenitors studied until now can be divided into two groups, one where the white dwarf explosion is triggered close to the center, and the other where the white dwarf explosion is triggered in the helium shell (see Livio 2001, for a review).

Two scenarios exist for close-to-center ignition. One is the double degenerate scenario, in which two $\mathrm{CO}$ white dwarfs in a binary system merge due to the angular momentum loss by gravitational wave radiation. If the system mass exceeds the Chandrasekhar mass, carbon must ignite, with the possible outcome of a thermonuclear explosion. Although there is observational evidence for double white dwarf systems (e.g., Koester et al. 2001; Karl et al. 2003), including one sdB star plus a $\mathrm{CO}$ white dwarf system which might result in a double white dwarf system having a total mass beyond the Chandrasekhar limit (Maxted et al. 2000), this scenario is criticized mainly by the fact that the merging of two white dwarfs is likely to end in an accretion-induced collapse rather than an SN explosion (Saio \& Nomoto 1985, 1998).

The currently favored scenario for close-to-center ignition is the single degenerate scenario, in which a $\mathrm{CO}$ white dwarf in a close binary system accretes hydrogen-rich or helium-rich 
matter from a non-degenerate companion at a relatively high accretion rate $\left(\sim 10^{-7} M_{\odot} / \mathrm{yr}\right)$. The super-soft X-ray sources, which are believed to consist of a non-degenerate star and a white dwarf, are promising observed counterparts of this scenario. The idea of steady nuclear burning due to a high accretion rate fits well to these sources (e.g., Li \& van den Heuvel 1997; Kahabka \& van den Heuvel 1997; Langer et al. 2000; Yoon \& Langer 2003). Symbiotic systems (e.g., Hachisu et al. 1999) and recurrent novae (e.g., Hachisu \& Kato 2001; Thoroughgood et al. 2001) may also belong to this scenario. However, it is still not clear whether this scenario can explain the observed SN Ia frequency since the accretion rates which allow steady burning are limited to a very narrow range. Furthermore, helium shell burning is generally found to be unstable: strong helium shell flashes might lead to strong mass loss by optically thick winds, or to a strong expansion of the white dwarf envelope to a red-giant phase which will result in a possible merging of the binary (e.g., Nomoto 1982a; Hachisu et al. 1996; Cassisi et al. 1998; Kato \& Hachisu 1999; Langer et al. 2002).

In the so called "Edge Lit Detonation" models, corresponding to sub-Chandrasekhar mass models, helium ignites at the bottom of a helium layer of a few tenths of a solar mass, accumulated on a $\mathrm{CO}$ white dwarf at a relatively low accretion rate ( $10^{-8} M_{\odot} /$ yr) (e.g., Nomoto 1982b; Limongi \& Tornambé 1991; Woosley \& Weaver 1994; Livne \& Arnett 1995). These models are favored in terms of statistics (e.g., Iben \& Tutukov 1991; Tout et al. 2001) but disfavored by the fact that the spectra and light curves produced by these models are not in agreement with observations (Höflich et al. 1996; Nugent et al. 1997; Pinto et al. 2001). Yoon \& Langer (2004b) showed that a helium detonation in such models may be avoided altogether due to a strong shear layer inside an accreting white dwarf.

Most previous studies of accreting white dwarfs as SN Ia progenitors have assumed spherical symmetry. However, in a close binary system which contains a white dwarf, an accretion disk is formed around the white dwarf. The matter coming from the accretion disk which is incorporated into the white dwarf carries high specific angular momentum, i.e. up to the value corresponding to Keplerian rotation at the white dwarf equator. Consequently, not only the accreted layer may be spinning very rapidly, but also the rest of the white dwarf, as angular momentum may be transported into the stellar interior (e.g., Ritter 1985; Langer et al. 2000).

Recent polarization observations provide evidence for the aspherical nature of SNe Ia (SN 1999by, Howell et al. 2001; SN 2001el, Wang et al. 2003). A plausible explanation for this asphericity may be a rapidly rotating progenitor, while clumpiness of supernova ejecta or binarity of the progenitors could be another possible cause for the observed polarization (Kasen et al. 2003). The observation that white dwarfs in Cataclysmic Variables rotate much faster than isolated ones (Sion 1999; Starrfield 2003) supports the idea that accreting white dwarfs are indeed spun up. In this picture, differential rotation inside the accreting white dwarf is inevitable, unless the angular momentum transport in the white dwarf is extremely efficient such that rigid body rotation may be maintained throughout the white dwarf interior (Yoon \& Langer 2004a). Rotationally induced instabilities such as the shear instability may lead to chemical mixing between the $\mathrm{CO}$ white dwarf material and the accreted matter. This effect has been discussed by several authors mainly with respect to the chemical enrichment of $\mathrm{CNO}$ and other heavier elements observed in nova ejecta (e.g., Kippenhahn \& Thomas 1978; MacDonald 1983; Livio \& Truran 1987; Fujimoto 1988; Fujimoto \& Iben 1997).

In this paper, we investigate the effects of rotation on the evolution of accreting white dwarfs in relation to the single degenerate Chandrasekhar mass scenario for SNe Ia progenitors. For this purpose, we carry out numerical simulations of helium burning white dwarfs considering high accretion rates ( $>10^{-7} M_{\odot} / \mathrm{yr}$ ) which allow steady shell burning for hydrogen or helium. Our discussions in this study are focused on the rotationally induced chemical mixing and its consequences for the behavior of the helium burning shell source. Comprehensive discussions of the rotational induced hydrodynamic instabilities and their role in the redistribution of angular momentum in accreting white dwarfs are given in a separate paper (Yoon \& Langer 2004a). Effects of rotation on sub-Chandrasekhar mass models, i.e., on accreting white dwarfs with relatively low accretion rates $\left(\sim 10^{-8} M_{\odot} / \mathrm{yr}\right)$, are discussed in Yoon \& Langer (2004b). Our computational method is introduced in Sect. 2, and the initial model and physical assumptions are discussed in Sect. 3. In Sect. 4 we present the evolution of various physical quantities of our white dwarf models. We discuss rotationally induced chemical mixing and the stability of shell burning in Sects. 5 and 6 respectively. Finally, we discuss our results in Sect. 7.

\section{Method}

The numerical models have been computed with a hydrodynamic stellar evolution code (cf., Langer 1998; Heger et al. 2000). Opacities are taken from Iglesias \& Rogers (1996). Changes in the chemical composition are computed using a nuclear network with more than 60 nuclear reactions, of which 31 reactions are relevant to helium burning such as $3 \alpha,{ }^{12} \mathrm{C}(\alpha, \gamma){ }^{16} \mathrm{O}(\alpha, \gamma){ }^{20} \mathrm{Ne}$ and ${ }^{20} \mathrm{Ne}(\alpha, \gamma){ }^{24} \mathrm{Mg}(\alpha, \gamma){ }^{28}$ Si reactions. For the ${ }^{12} \mathrm{C}(\alpha, \gamma){ }^{16} \mathrm{O}$ reaction rate, which has an important role in our study, we use the rate of Caughlan et al. (1985) reduced by a factor of 0.63 , as suggested by Weaver \& Woosley (1993).

The effect of the centrifugal force on the stellar structure, and rotationally induced transport of angular momentum and chemical species are treated in a one-dimensional approximation (Heger et al. 2000). We consider rotationally induced transport processes such as Eddington-Sweet circulations, the Goldreich-Schubert-Fricke instability, and the dynamical and secular shear instability, by solving a non-linear diffusion equation, with diffusion coefficients for each of the instabilities computed as in Heger et al. (2000) and Yoon \& Langer (2004a). The viscous dissipation of the rotational energy is also considered as described by Yoon \& Langer (2004a). More details about the numerical methods are given in Yoon \& Langer (2004a).

A comparison of our method with previously used ones for rotating white dwarf models has already been given in 
Yoon \& Langer (2004a) in detail. Here we give some additional comments on the rotation physics in relation to heliumaccreting white dwarfs.

Our approach to include rotation is based on the method given by Kippenhahn \& Thomas (1970), with the mass shells of a star defined by isobars instead of spherical shells. In describing effects of rotationally induced chemical mixing, the chemical abundances are assumed to be constant on isobars. This has been justified by theoretical achievements of Zahn (1975), Chaboyer \& Zahn (1992) and Zahn (1992), who showed that anisotropic turbulence acts much more efficiently in the horizontal than in the vertical direction. Shellular rotation and chemical homogeneity on isobars can thus be achieved (Meynet \& Maeder 1997), enabling us to use a one dimensional approximation (see also Maeder 2003).

Our assumption of shellular rotation may be questionable for helium-accreting white dwarfs, on two accounts. Kippenhahn \& Thomas (1978) argued that the accreted matter cannot spread over the whole surface of the white dwarf but rather forms an accretion belt, since the angular momentum transport time scale based on the electron viscosity is longer than the accretion time scale. However, calculations by Sparks \& Kutter (1987) failed to obtain classical nova outbursts when the prescription of accretion belts was employed. Livio \& Truran (1987) argued that if turbulent viscosity is used, the angular momentum transport becomes much more efficient than discussed by Kippenhahn \& Thomas. They concluded that the accreted matter can spread over the entire star with the consequence that mass accretion onto white dwarfs may result in nova outbursts, which was not possible in the accretion belt prescription due to the too strong centrifugal force at the equator. A local stability analysis by MacDonald (1983) and a theoretical study by Fujimoto (1988) also concluded that the accreted matter will not remain confined to the equatorial belt due to efficient angular momentum transport toward the poles. We therefore conclude that the assumption of shellular rotation employed in our study may still be well-grounded, at least in the non-degenerate helium envelope, although multidimensional simulations are ultimately required for its justification (cf. Hujeirat 1995).

Secondly, as degenerate matter is barotropic, perturbations which create $\partial j / \partial z \neq 0$, with $j$ as specific angular momentum and $z$ being the distance to the equatorial plane, are eliminated on a short time scale. Thus, cylindrical rotation may be enforced in the inner CO core (e.g., Kippenhahn \& Möllenhoff 1974; Durisen 1977). This means that accreting white dwarfs may consist of two different rotation laws: shellular in the nondegenerate envelope and cylindrical rotation in the degenerate core. However, our numerical models can still represent the cylindrically rotating degenerate inner core to some degree, since most of the total angular momentum is confined at the equatorial plane in both the shellular and the cylindrical cases. It is harder to estimate the angular momentum transport efficiency in the semidegenerate transition layer, as non-barotropic perturbations in the degenerate core may induce dynamical meridional flows (Kippenhahn \& Möllenhoff 1974; Müller 2003, private communication). Since it is poorly known how these meridional flows may affect the angular momentum redistribution, we have to leave the detailed investigation of this effect to future work.

Finally, the accuracy of our results for very rapid rotation is limited due to the one-dimensional approximation. In computing the effective gravitational potential in a rotating star, the potential is expanded in terms of spherical harmonics, of which we only consider terms up to the second order (Kippenhahn \& Thomas 1970; Endal \& Sofia 1976). This method can reproduce the shapes of rigidly rotating polytropes accurately up to a rotation rate of about $60 \%$ of the critical value, corresponding to correction factors of $f_{P} \simeq 0.75$ and $f_{T} \simeq 0.95$ in the stellar structure equations (Fliegner 1993; Heger et al. 2000). We therefore limit these factors to the quoted values, with the consequence that we underestimate the effect of the centrifugal force in layers that rotate more rapidly than about $60 \%$ critical. We shall see below that this affects only a very small fraction of our stellar models. However, as the surface layers are always very close to critical rotation, the stellar radius of our models may be somewhat underestimated. A better description of the equilibrium structure of rapidly rotating stars which may deviate significantly from spheres requires multi-dimensional techniques such as the self-consistent field method (Ostriker \& Mark 1968; Hachisu 1986). In addition, since our models are rotationally symmetric, three-dimensional effects such as triaxial deformation cannot be described with our numerical code.

\section{Physical assumptions}

We begin with a hot carbon-oxygen white dwarf of $\log L_{\mathrm{S}} / L_{\odot}=3.379$ to avoid the numerical difficulty of a strong initial helium shell flash. The initial mass of our model is $0.998 M_{\odot}$. The central temperature and density are initially such that $T_{\mathrm{c}}=2.288 \times 10^{8} \mathrm{~K}$ and $\rho_{\mathrm{c}}=1.64 \times 10^{6} \mathrm{~g} \mathrm{~cm}^{-3}$, respectively. Although in reality white dwarfs may be much colder when mass accretion starts, the thermal evolution of the white dwarf core is not influenced by the different initial conditions due to the self heating of white dwarfs (cf. Yoon \& Langer 2003). Since observations (Heber et al. 1997; Koester et al. 1998; Kawaler 2003) and stellar evolution models (Langer et al. 1999) find small spin rates of isolated white dwarfs, we adopt a slow and rigid rotation in our initial model: the initial rotation velocity at the white dwarf equator is set to $1.0 \mathrm{~km} \mathrm{~s}^{-1}$.

We consider 4 different accretion rates: $2 \times 10^{-7}, 3 \times 10^{-7}$, $5 \times 10^{-7}$ and $10^{-6} M_{\odot} / y r$. The accreted matter is assumed to be helium-rich with $Y=0.98$ and $Z=0.02$. The abundances for heavier elements are chosen to be the same as in a helium core which results from core hydrogen burning in a main sequence star through the CNO cycle.

The specific angular momentum of the accreted matter is assumed to have the local Keplerian value, as most white dwarfs with a non-degenerate companion are believed to accrete matter through an accretion disk. However, analytic considerations can show this to cause an angular momentum problem. Assuming rigid rotation and a usual white dwarf mass-radius relation, the white dwarf reaches overcritical rotation long before reaching the Chandrasekhar limit (Livio \& Pringle 1998; Langer et al. 2000). For finite angular momentum redistribution times, as realized by our preliminary 
Table 1. Properties of the computed models. The first column denotes the sequence number, where "NR", "R" and "RT" denote the non-rotating models and rotating models with $f_{\mathrm{c}}=1 / 30$ and $1 / 3$ respectively. The second column gives the accretion rate in units of $10^{-7} M_{\odot} / \mathrm{yr}$. The third column denotes the factor $f_{\mathrm{c}}$ for the chemical mixing efficiency in rotating models. Columns 4-7 give mass, central density, central temperature and $E_{\mathrm{rot}} /\left|E_{\mathrm{grav}}\right|$ of the last computed model of each evolutionary sequence. The eighth column gives the amount of accreted material until the onset of the helium shell instability. The column with $L_{\text {Edd }}$ indicates whether the last computed model reached the Eddington limit or not. The last column gives the total number of the computed models for each sequence.

\begin{tabular}{|c|c|c|c|c|c|c|c|c|c|}
\hline No. & $\begin{array}{c}\dot{M} \\
{\left[10^{-7} M_{\odot} / \mathrm{yr}\right]}\end{array}$ & $f_{\mathrm{c}}$ & $\begin{array}{l}M_{\text {last }} \\
{\left[M_{\odot}\right]} \\
\end{array}$ & $\begin{array}{c}\rho_{\mathrm{c}, \text { last }} \\
{\left[10^{8} \mathrm{~g} / \mathrm{cm}^{3}\right]}\end{array}$ & $\begin{array}{c}T_{\text {c,last }} \\
{\left[10^{8} \mathrm{~K}\right]}\end{array}$ & $\left(E_{\text {rot }} /\left|E_{\text {grav }}\right|\right)_{\text {last }}$ & $\begin{array}{c}\Delta M \\
{\left[M_{\odot}\right]} \\
\end{array}$ & $L_{\mathrm{Edd}}$ & No. of models \\
\hline$\overline{\text { NR1 }}$ & 2.0 & - & 1.0026 & 0.25 & 1.45 & - & 0.00 & Yes & 4342 \\
\hline NR2 & 3.0 & - & 1.0042 & 0.25 & 1.45 & - & 0.00 & Yes & 5164 \\
\hline NR3 & 5.0 & - & 1.0130 & 0.28 & 1.37 & - & 0.00 & Yes & 10324 \\
\hline NR4 & 10 & - & 1.1984 & 1.39 & 1.44 & - & 0.03 & No & 383637 \\
\hline R1 & 2.0 & $1 / 30$ & 1.0074 & 0.28 & 1.25 & 0.0018 & 0.00 & Yes & 18021 \\
\hline $\mathrm{R} 2$ & 3.0 & $1 / 30$ & 1.0224 & 0.32 & 1.17 & 0.0025 & 0.00 & Yes & 28449 \\
\hline $\mathrm{R} 3$ & 5.0 & $1 / 30$ & 1.2241 & 1.05 & 1.21 & 0.0197 & 0.14 & No & 180004 \\
\hline $\mathrm{R} 4$ & 10 & $1 / 30$ & 1.4773 & 5.14 & 1.72 & 0.0578 & 0.48 & No & 490026 \\
\hline RT1 & 2.0 & $1 / 3$ & 1.0665 & 0.42 & 0.99 & 0.0081 & 0.01 & No & 174270 \\
\hline RT2 & 3.0 & $1 / 3$ & 1.1537 & 0.77 & 1.22 & 0.0080 & 0.01 & No & 212730 \\
\hline
\end{tabular}

calculations (Yoon \& Langer 2002), overcritical rotation is even reached earlier.

Livio \& Pringle (1998) suggested nova explosions as a possible mechanism for angular momentum loss to explain the white dwarf rotation velocity in typical CV systems. However, within the single degenerate SNe Ia progenitor scenario in which the white dwarf must grow to the Chandrasekhar limit by mass accretion, we need another mechanism to solve the angular momentum problem. To prevent the white dwarf from rotating over-critically, in our calculations, we limited the angular momentum gain in a simple way as follow. If the surface velocity at the white dwarf equator is below the Keplerian value, the specific angular momentum of the accreted matter is assumed to have the Keplerian value. Otherwise, no angular momentum is allowed to be accreted onto the white dwarf while mass is still accreted:

$j($ accreted matter $)= \begin{cases}v_{\text {Kepler }} R_{\mathrm{WD}} & \text { if } v_{\mathrm{s}}<v_{\text {Kepler }} \\ 0 & \text { if } v_{\mathrm{s}}=v_{\text {Kepler }}\end{cases}$

where $v_{\text {Kepler }}=\sqrt{G M_{\mathrm{WD}} / R_{\mathrm{WD}}}$, and $v_{\mathrm{s}}$ is the surface rotation velocity of the accreting white dwarf at its equator. This assumption may be justified by considerations of Paczyński (1991) and Popham \& Narayan (1991) to allow angular momentum transport from the white dwarf surface through the boundary layer into the accretion disk by turbulent viscosity when the spin rate of the white dwarf reaches the Keplerian value, without preventing efficient mass accretion.

As discussed in Heger et al. (2000), the contribution of the rotationally induced instabilities to the chemical diffusion is considered by introducing a factor $f_{\mathrm{c}} \in[0,1]$, which describes the ratio of the chemical diffusion coefficient to turbulent viscosity. Heger et al. (2000) and Heger \& Langer (2000) chose a value of $f_{\mathrm{c}}=1 / 30$ following Chaboyer \& Zahn (1992) (see also Pinsonneault et al. 1989). In the present study, we consider two different values for $f_{\mathrm{c}}$, i.e., $1 / 30$ and $1 / 3$ (see Table 1 ), in order to investigate how the strength of chemical mixing affects the nuclear shell burning.
For a comparative study, rotating and non-rotating models with the same initial conditions have been investigated. The properties of all computed models are summarized in Table 1.

\section{Evolution}

The results of our calculations are summarized in Table 1. For sequences NR1, NR2, NR3, R1 and R2, we followed the evolution until the helium shell flashes become strong enough to drive the surface luminosity to the Eddington limit. For the remainder, calculations are stopped after a large number of models has been calculated (cf. Table 1).

\subsection{Helium shell burning and luminosity}

In Fig. 1, the nuclear luminosity due to the helium burning in the sequences with $\dot{M}=2 \times 10^{-7} M_{\odot} / \mathrm{yr}$ (NR1, R1 and RT1) is shown as a function of the total mass of the white dwarf. In sequence NR1, helium shell burning is not stable but undergoes thermal pulses, which are driven by the thin shell instability (e.g., Schwarzshild \& Härm 1965; Weigert 1966). It is well known that this phenomenon occurs also in intermediate mass stars in their thermally pulsating asymptotic giant branch (TP-AGB) phase (e.g., Iben \& Renzini 1983). The thermal pulses in sequence NR1 become stronger as the white dwarf accretes more matter (cf. Fujimoto \& Sugimoto 1979). Finally, the shell flash at the sixth pulse drives the surface luminosity to the Eddington limit when $M_{\mathrm{WD}} \simeq 1.002 M_{\odot}$ (cf. Fig. 2), at which point we stop the calculation. Perhaps, the white dwarf would experience a radiation-driven optically thick outflow at this stage, which would reduce the mass accumulation efficiency (Kato \& Hachisu 1999). It is beyond the purpose of this paper to consider the ensuing evolution in detail.

In the rotating sequence $\mathrm{R} 1$ with the same accretion rate, thermal pulses become significantly weaker than in the nonrotating case. For instance, the helium shell source reaches $\log L_{\mathrm{N}} / L_{\odot} \simeq 6.5$ in the non-rotating case when the white dwarf has accreted about $0.004 M_{\odot}$, while the rotating sequence only 

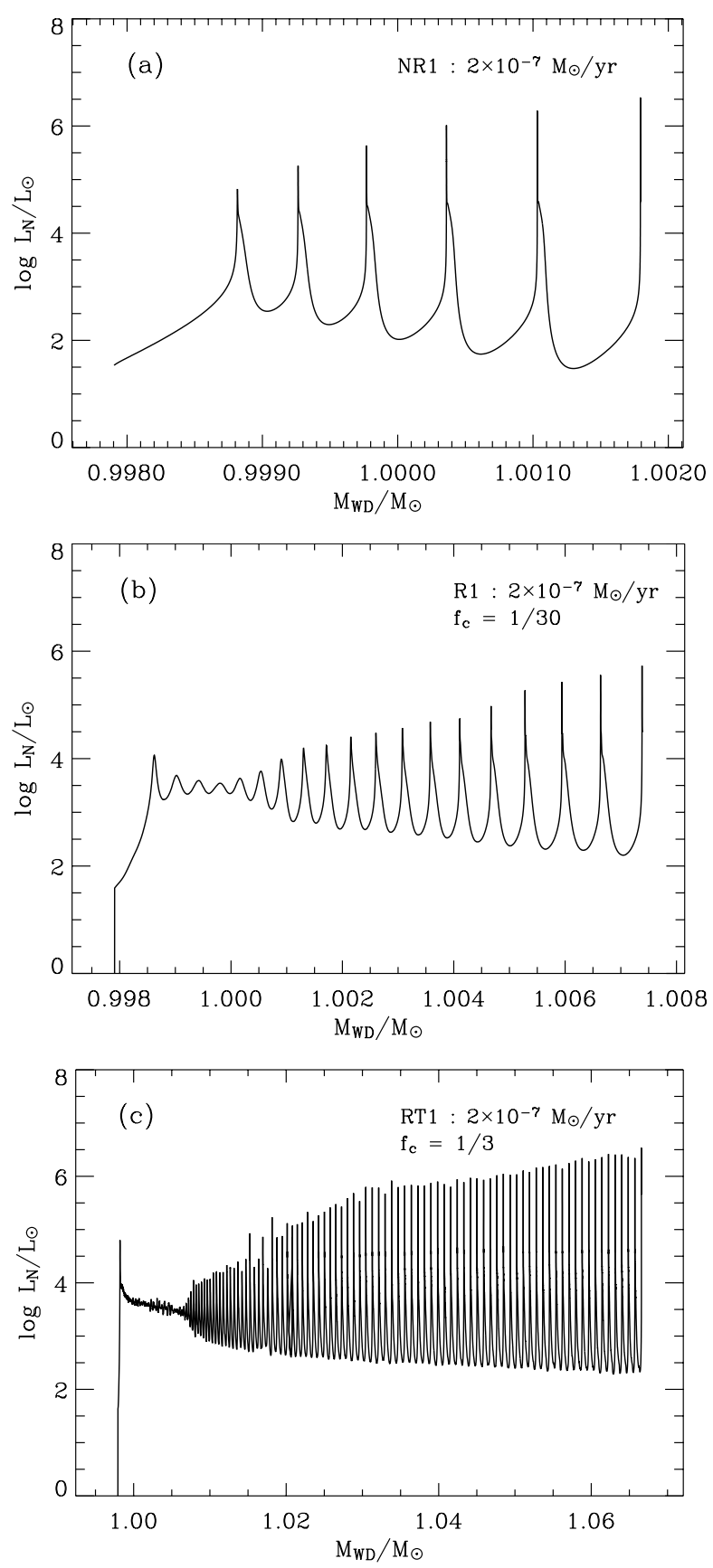

Fig. 1. Evolution of nuclear luminosity due to helium burning in the helium-accreting white dwarf models with $\dot{M}=2 \times 10^{-7} M_{\odot} /$ yr. The box in panel a) gives the result from the non-rotating case (NR1) while the results from the rotating models with $f_{\mathrm{c}}=1 / 30(\mathrm{R} 1)$ and $f_{\mathrm{c}}=$ $1 / 3$ (RT1) are shown in panels b) and c). The abscissa denotes the total mass of the white dwarf, which serves as a measure of time.

reaches $\log L_{\mathrm{N}} / L_{\odot} \simeq 4.0$ at the same epoch (see Fig. 1). As a consequence, the white dwarf can accrete more mass $\left(\Delta M_{\mathrm{acc}} \simeq\right.$ $\left.0.0096 M_{\odot}\right)$ than in sequence NR1 $\left(\Delta M_{\text {acc }} \simeq 0.0039 M_{\odot}\right)$ before the surface luminosity reaches the Eddington limit. In the HR diagram (Fig. 2), the rotating sequence is located at lower effective temperatures than the non-rotating sequence, since the white dwarf envelope is expanded by the centrifugal force.

The helium shell source becomes more stable with stronger chemical mixing. Results of sequence RT1 in Fig. 1c, where
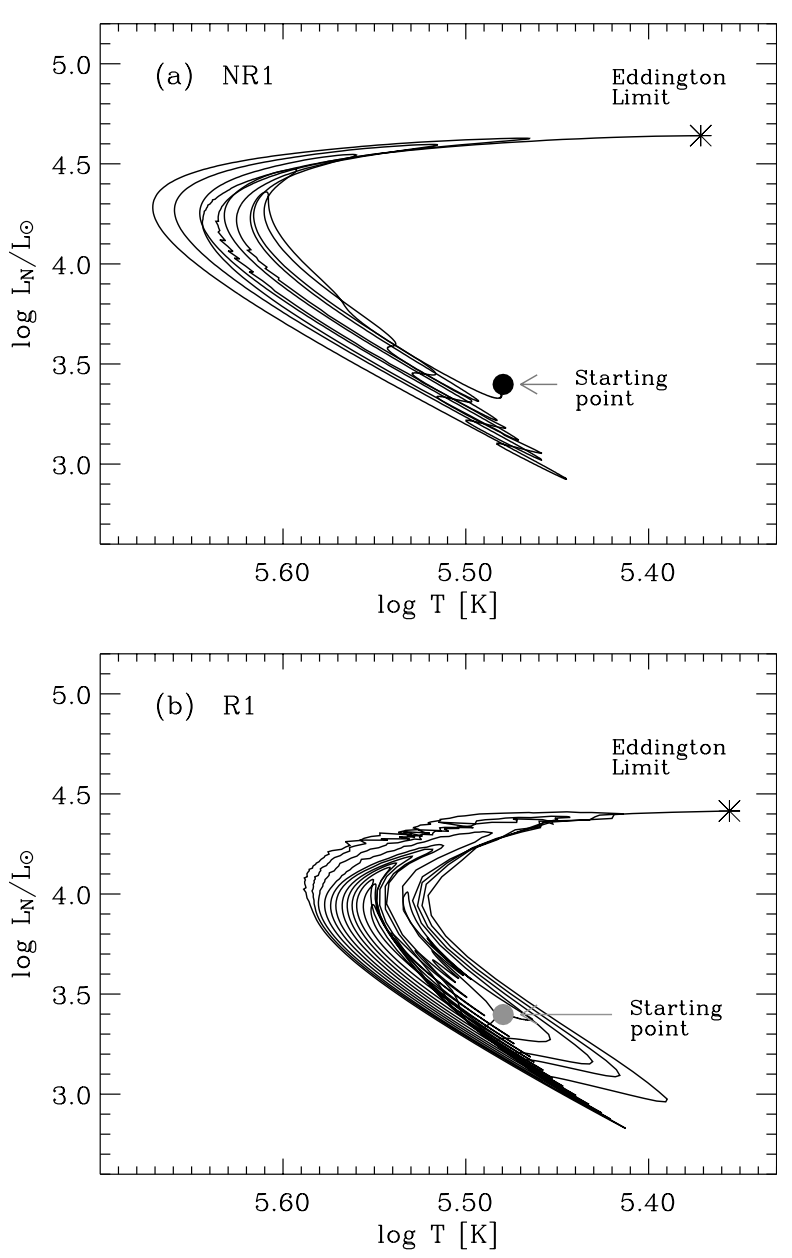

Fig. 2. Evolution of the white dwarf in the sequences NR1 a) and R1 b) in the Hertzprung-Russell diagram. The starting point is marked by a filled circle. The endapoint, where the surface luminosity reaches the Eddington limit, is marked by an asterisk for each case. The raggedness which appears in some parts of the evolutionary tracks is due to the limited number of digits in the numerical outputs.

the chemical mixing is 10 times stronger than in sequence $\mathrm{R} 1$, show that the helium is burned more or less steadily until $M_{\mathrm{WD}} \simeq 1.01 M_{\odot}$. More than 100 pulses were followed thereafter, but the surface luminosity still did not reach the Eddington limit.

The evolution of sequence NR2 $\left(\dot{M}=3 \times 10^{-7} M_{\odot} / \mathrm{yr}\right)$ shown in Fig. 3 is similar to that of NR1, only the white dwarf reaches the Eddington limit somewhat later, i.e., when $M_{\mathrm{WD}} \simeq 1.004 M_{\odot}$. The thermal pulses are weakened in the corresponding rotating sequence with $f_{\mathrm{c}}=1 / 30$ (R2, Fig. 3b), and about 4 times more mass could be accreted in the white dwarf of sequence $\mathrm{R} 2$ before the Eddington limit was reached. With a stronger chemical mixing ( $f_{\mathrm{c}}=1 / 3$, RT2, Fig. 3c), steady helium shell burning continues until $M_{\mathrm{WD}} \simeq 1.01 M_{\odot}$, and weak thermal pulses with $\log L_{\mathrm{N}} / L_{\odot} \lesssim 5.0$ follow. The evolution of this sequence was stopped when $M_{\mathrm{WD}} \simeq 1.15 M_{\odot}$, as the number of thermal pulses before reaching the Chandrasekhar mass would exceed $10^{4}$.

The stabilizing effect of rotation appears more prominently at $\dot{M}=5 \times 10^{-7} M_{\odot} /$ yr. In the non-rotating case (NR3), the 

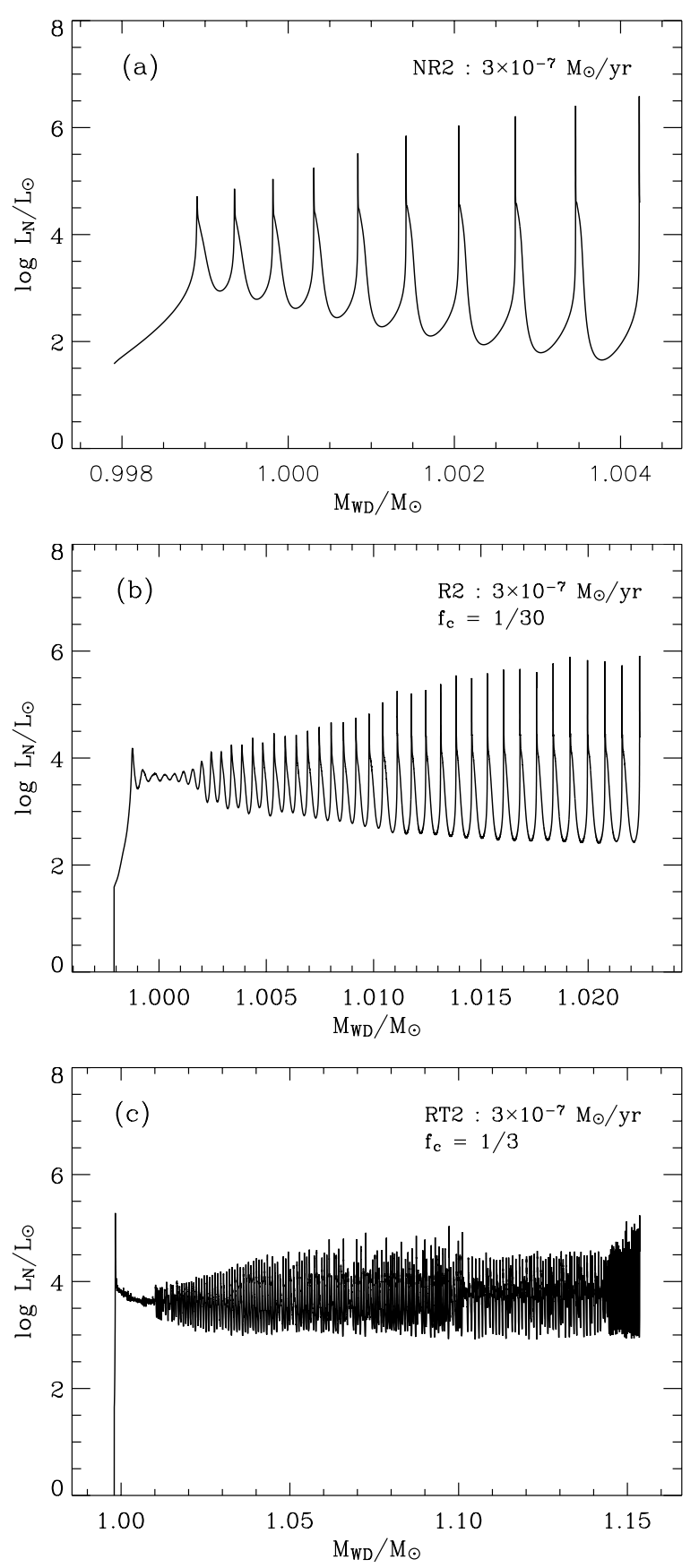

Fig. 3. Same as in Fig. 1 but with $\dot{M}=3 \times 10^{-7} M_{\odot} / \mathrm{yr}(\mathrm{NR} 2$ a), R2 b) and RT2 c)).

helium shell burning is still unstable even with such a high accretion rate, as shown in Fig. 4a, and the white dwarf reaches the Eddington limit when $M_{\mathrm{WD}} \simeq 1.013 M_{\odot}$. In the corresponding rotating sequence (R3), very weak thermal pulses appear when $M_{\mathrm{WD}} \simeq 1.005 M_{\odot}$, but are suppressed when $M_{\mathrm{WD}} \gtrsim 1.02 M_{\odot}$. Steady helium shell burning continues until $M_{\mathrm{WD}} \simeq 1.14 M_{\odot}$, from which point the shell source becomes again unstable. The thermal pulses shown in Fig. 4b look like noise, but actually each pulse is resolved by about $10^{3}$ models.

With an accretion rate of $\dot{M}=10^{-6} M_{\odot} / \mathrm{yr}$, the nonrotating white dwarf undergoes unstable helium shell burning after $M_{\mathrm{WD}} \simeq 1.03 M_{\odot}(\mathrm{NR} 4$, Fig. $4 \mathrm{c})$. We have followed the evolution until $M_{\mathrm{WD}}=1.2 M_{\odot}$ and find that thermal pulses do not become strong enough to lead the white dwarf to the Eddington limit. On the other hand, in the rotating sequence R4 (Fig. 4d), the white dwarf can grow up to $M_{\mathrm{WD}} \simeq 1.48 M_{\odot}$ without encountering significant thermal pulses.

The evolution of the white dwarf of sequence R4 in HR diagram is shown in Fig. 5. The surface luminosity remains nearly constant at $\log L_{\mathrm{s}} / L_{\odot} \simeq 4.2$, while the surface temperature increases from $\log T=5.46$ to $\log T=5.88$. Therefore, the white dwarf will appear as a super-soft X-ray source throughout the accretion phase (e.g., Kahabka \& van den Heuvel 1997; Greiner 2000).

The results presented in this section show that the helium shell source becomes more stable with rotation, and that the stronger the chemical mixing the more stable the shell burning becomes. We discuss the physical reasons for this behavior in Sect. 6.

\subsection{White dwarf spin and angular momentum transport}

According to Yoon \& Langer (2004a), who considered accretion of carbon-oxygen rich matter, non-magnetic accreting white dwarfs with $\dot{M} \gtrsim 10^{-7} M_{\odot} /$ yr show the following features in relation to the angular momentum redistribution. a) Eddington-Sweet circulations, the secular shear instability, and the GSF instability are dominating the angular momentum redistribution in the non-degenerate outer envelope, while the dynamical shear instability is the most important in the degenerate core. b) The degree of differential rotation in the degenerate core remains strong throughout the evolution, with a shear strength near the threshold value for the onset of the dynamical shear instability. c) The resulting differential rotation changes the white dwarf structure such that the center does not reach carbon ignition even when the white dwarf reaches the Chandrasekhar limit $\left(\sim 1.4 M_{\odot}\right)$. d) More than $60 \%$ of the angular momentum of the accreted matter is rejected because of the condition that the white dwarf should not gain angular momentum when its surface rotates critically (Eq. (1)).

It is found that all these features remain the same in the present study, where helium accretion is considered. Figure 6 shows angular velocity profiles throughout white dwarf models of sequence RT1, R3 and R4, at various times, where the angular velocity is given in units of the local Keplerian value (i.e., $\left.\omega_{\text {Kep }}=\sqrt{G M_{r} / r^{3}}\right)$. Note that the spin rate remains well below the local Keplerian value throughout the whole star in all cases.

The white dwarf model with $M_{\mathrm{WD}} \simeq 1.4 M_{\odot}$ in sequence R4 has a central density of $3.54 \times 10^{8} \mathrm{~g} / \mathrm{cm}^{3}$, which is still far from the carbon ignition density. (cf. Ostriker \& Bodenheimer 1968; Durisen 1975; Durisen \& Imamura 1981). As shown in the mentioned papers, a differentially rotating white dwarf can be dynamically stable up to $\sim 4.0 M_{\odot}$. As discussed in Yoon \& Langer (2004a), this suggests that the secular instability to the gravitational wave radiation reaction, which is often called as the "CFS instability" (Chandrasekhar 1970; Friedman \& Schutz 1978), might be important for the final fate of accreting white dwarfs near or above the Chandrasekhar limit. The ratio 

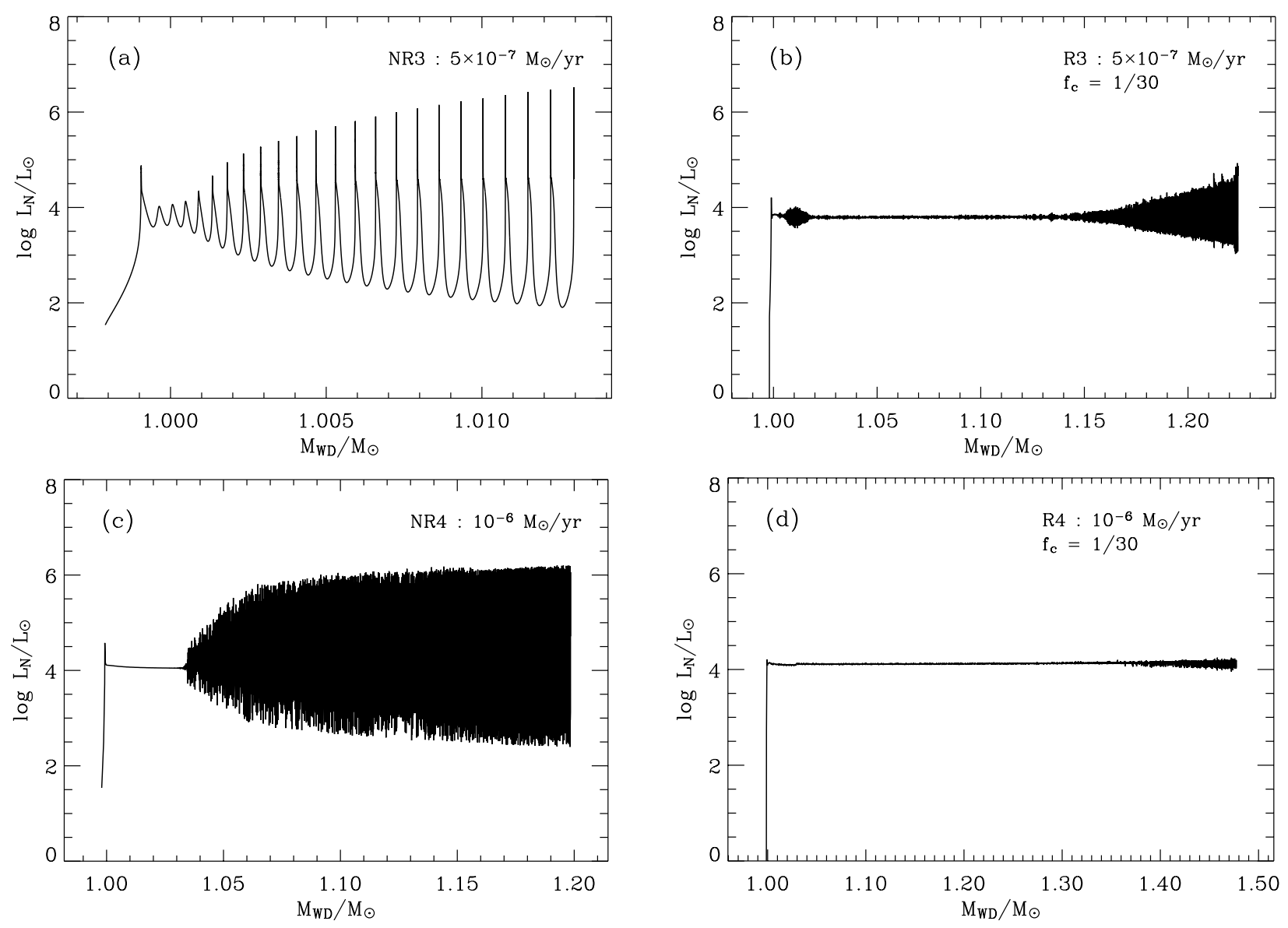

Fig. 4. Evolution of nuclear luminosity due to helium burning in the helium-accreting white dwarf models for $\dot{M}=5 \times 10^{-7} M_{\odot} / \mathrm{yr}$ (NR3 a) and R3 b)) and $\dot{M}=10^{-6} M_{\odot} / \mathrm{yr}(\mathrm{NR} 4$ c) and R4 d)). The abscissa denotes the total mass of the white dwarf, which serves as a measure of time. Note that what appears to look like noise in the plots for R3 and NR4 corresponds to many successive thermal pulses, each as well resolved in time (i.e., by roughly $10^{3}$ time steps) as those in the plots in Figs. 1 and 3.

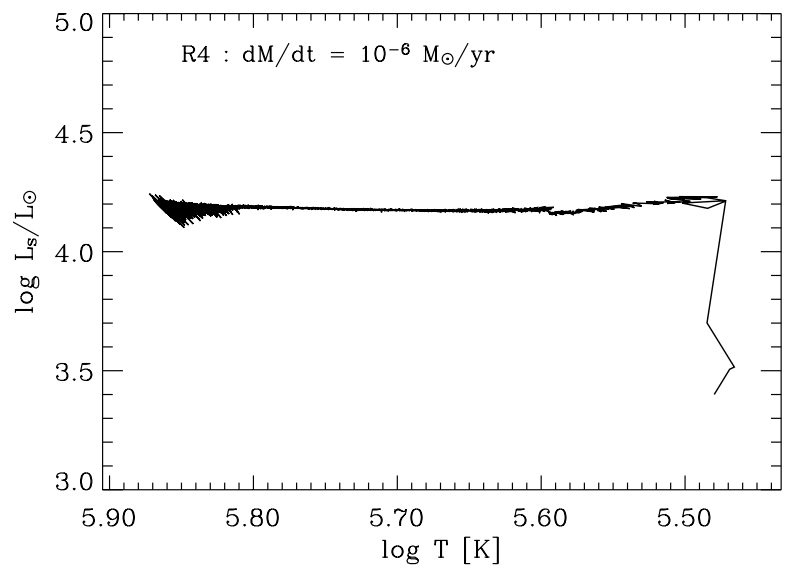

Fig. 5. Evolution of the white dwarf in sequence R4 in the Hertzsprung-Russell diagram. The region with $\log L_{\mathrm{s}} / L_{\odot}=3.4 \ldots 4.2$ and $\log T=5.46 \ldots 5.48$ represents the epoch from the onset of helium accretion to the first mild helium shell flash (cf. Fig. 4d).

of the rotational energy to the gravitational energy ( $\left.E_{\text {rot }} /\left|E_{\text {grav }}\right|\right)$, which determines the growth time scale of the CFS instability via the bar-mode or the $r$-mode (see Yoon \& Langer 2004a), remains smaller than 0.1 in the white dwarf models of sequence $\mathrm{R} 4$, and the last model $\left(M_{\mathrm{WD}}=1.48 M_{\odot}\right)$ has
$E_{\text {rot }} /\left|E_{\text {grav }}\right|=0.06$. Since in white dwarfs with strong differential rotation $E_{\text {rot }} /\left|E_{\text {grav }}\right| \gtrsim 0.1$ is required for the onset of the bar-mode instability (Imamura et al. 1995), only the $r$-mode may be relevant at this point. The expected growth time of the $r$-mode instability in this model is about $1.1 \times 10^{5} \mathrm{yr}$ (see Yoon \& Langer 2004a; cf. Lindblom 1999). We refer to Yoon \& Langer (2004a) for a detailed discussion of the final fate of rapidly rotating massive white dwarfs.

\section{Chemical mixing and nucleosynthesis}

The mixing of $\alpha$-particles into the CO-core and of carbon into the helium envelope has consequences for the helium burning shell source. Here, we discuss the resulting changes in chemical structure of the white dwarf, while in the next section we analyze how rotation and rotationally induced chemical mixing affects the stability properties of the shell source.

Figure 7 shows the chemical structure and the nuclear energy generation rate in the helium burning shell in the white dwarf models of sequences R4 and NR4 when $M_{\mathrm{WD}}=$ $1.0305 M_{\odot}$. The helium layer in the rotating model extends farther into the $\mathrm{CO}$ core than in the non-rotating case, due to the rotationally induced chemical mixing. This renders the 

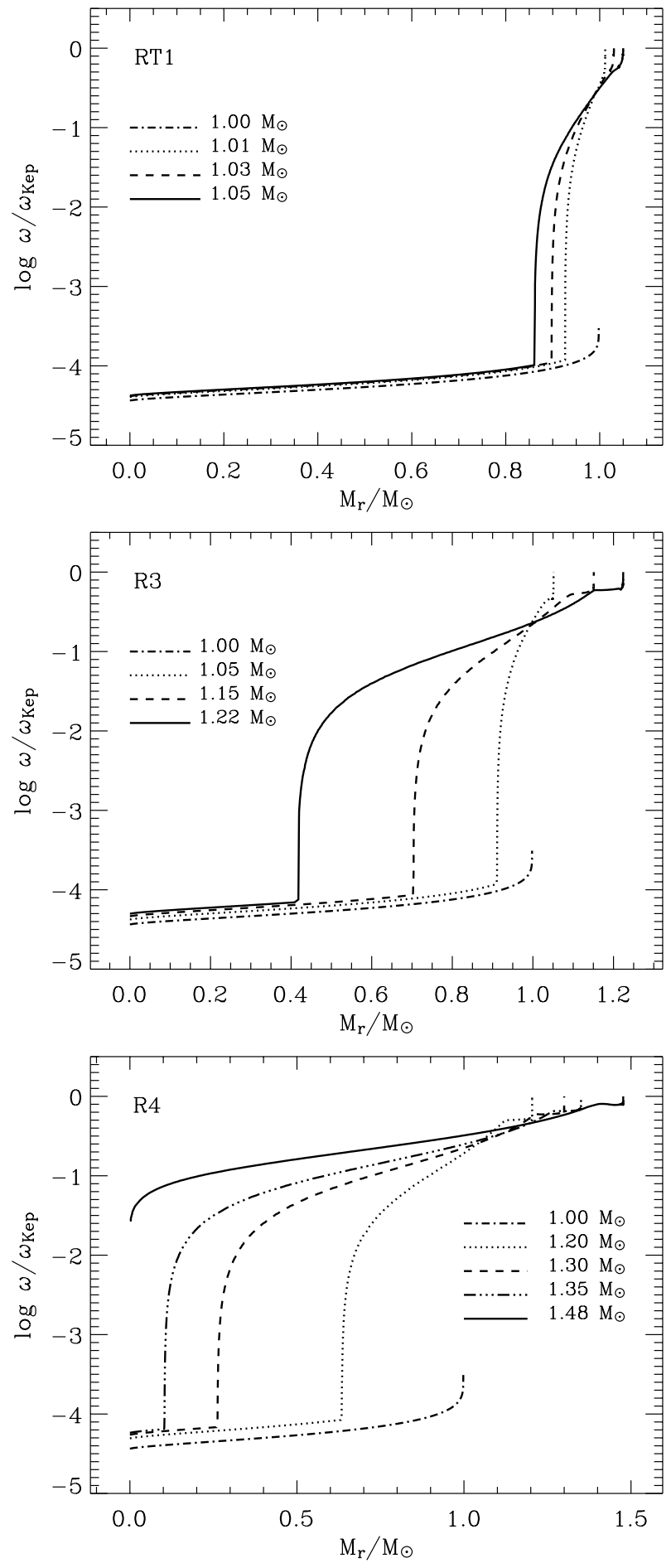

Fig. 6. Spin velocity at different white dwarf masses, normalized to the local Keplerian value, as a function of the mass coordinate for RT1, R3 and R4.

${ }^{12} \mathrm{C}(\alpha, \gamma){ }^{16} \mathrm{O}$ reaction more active in the rotating model, with two main consequences.

Firstly, the contribution of the ${ }^{12} \mathrm{C}(\alpha, \gamma){ }^{16} \mathrm{O}$ reaction to the total energy production is considerably increased. In the given models in Fig. 7, the rotating model gives $\log L_{3 \alpha} / L_{\odot}=3.938$ and $\log L_{12} \mathrm{C}(\alpha, \gamma) / L_{\odot}=3.562$, while the non-rotating model gives $\log L_{3 \alpha} / L_{\odot}=3.963$ and $\log L_{12} \mathrm{C}(\alpha, \gamma) / L_{\odot}=3.338$, where $L_{3 \alpha}$ and $L_{12} \mathrm{C}(\alpha, \gamma)$ are the integrated nuclear energy generation
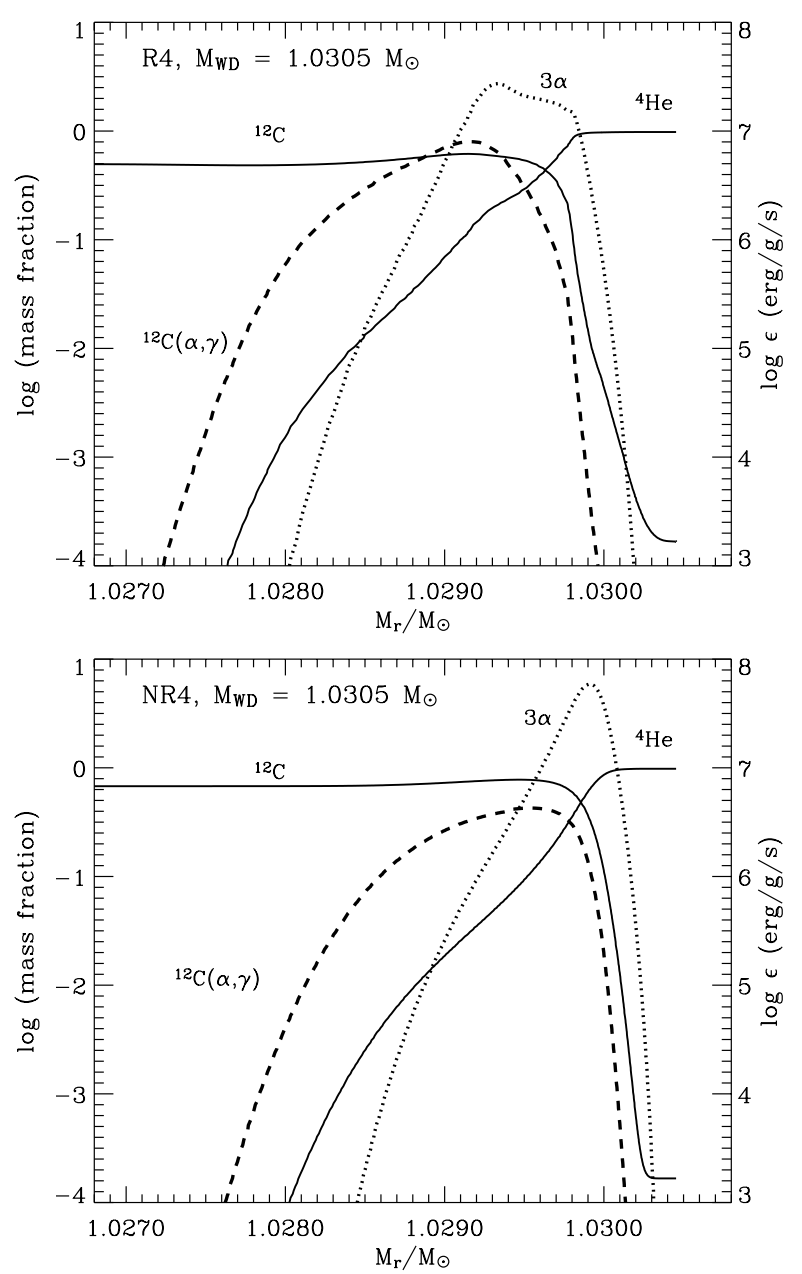

Fig. 7. Logarithm of the mass fractions of helium and carbon as a function of the mass coordinate (solid line), in the white dwarf models of sequences NR4 (upper panel) and R4 (lower panel) at a time when $M_{\mathrm{WD}}=1.0305 M_{\odot}$. The dotted and dashed lines denote the corresponding energy generation rate (right scale) for the $3 \alpha$ and ${ }^{12} \mathrm{C}(\alpha, \gamma)$ reactions respectively.

rates over the shell source, due to the triple alpha reaction and the ${ }^{12} \mathrm{C}(\alpha, \gamma){ }^{16} \mathrm{O}$ reaction, respectively. The $3 \alpha$ reaction is weaker in the rotating model than in the non-rotating one due to a lower density in the helium envelope (cf. Fig. 9) and due to a smaller mass fraction of helium at the peak of the nuclear energy generation rate caused by rotationally induced mixing (Fig. 7). However, the increase of the energy generation by the ${ }^{12} \mathrm{C}(\alpha, \gamma){ }^{16} \mathrm{O}$ reaction is more important on the whole, leading to a higher nuclear luminosity in the rotating model $\left(\log L_{\mathrm{N}} / L_{\odot}=4.094\right)$ than in the corresponding non-rotating case $\left(\log L_{N} / L_{\odot}=4.060\right)$, for the models given in Fig. 7 . Furthermore, the shell source is hotter in the rotating model $\left[T_{\text {shell }} /\left(10^{8} \mathrm{~K}\right)=2.16\right]$ than in the comparable non-rotating model $\left[T_{\text {shell }} /\left(10^{8} \mathrm{~K}\right)=2.03\right]$, which affects its stability as discussed in Sect. 6.

Secondly, the enhanced ${ }^{12} \mathrm{C}(\alpha, \gamma)$-efficiency results in an increased oxygen abundance in the ashes of the helium burning shell, at the expense of carbon. Figure 8 shows the carbon mass fraction throughout the white dwarf for the last computed models of sequences NR3 and R3. In the non-rotating 


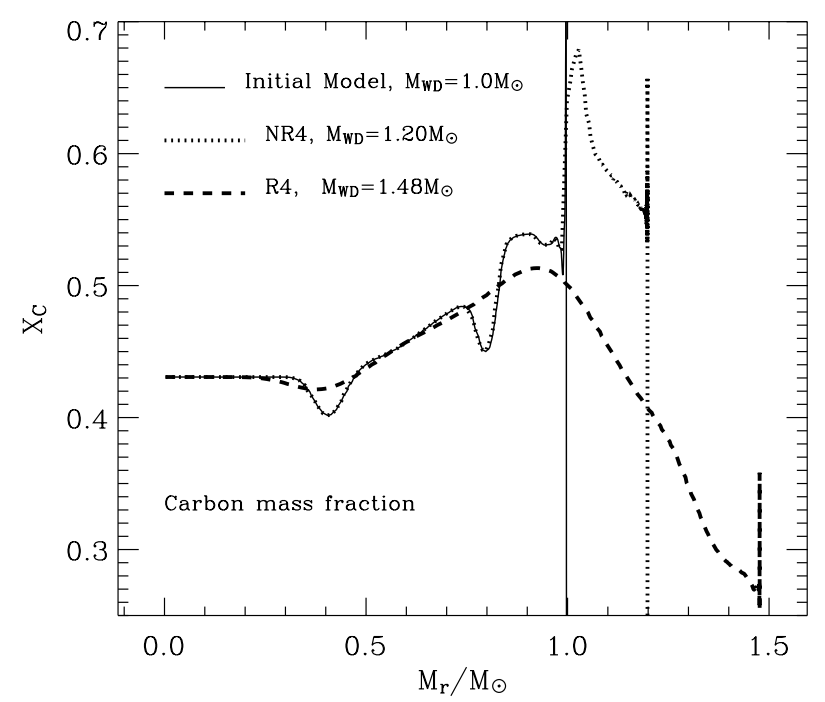

Fig. 8. Carbon mass fraction as a function of the mass coordinate for the last computed models of sequences R4 (dashed line) and NR4 (dotted line). The thin solid line corresponds to the initial model. Note that the mass of the helium envelope is only of the order of $10^{-4} M_{\odot}$, which is not resolved in this figure. The sharp peak in the carbon abundance near the surface in each white dwarf model is due to the active $3 \alpha$ reaction at the bottom of the helium envelope (cf. Fig. 7).

model, the carbon mass fraction produced by the shell source reaches almost $X_{\mathrm{C}} \simeq 0.68$ at a white dwarf mass of $1.03 M_{\odot}$. At this time, the helium shell source becomes unstable and develops thermal pulses. As the layers at and above the shell source become convectively unstable during each pulse, the resulting carbon abundance is reduced. The convective mixing becomes more and more significant as thermal pulses become stronger (cf. Fig. 4c), which is reflected in the drop of $X_{\mathrm{C}}$ from about 0.68 to 0.55 in the range $1.03 M_{\odot} \lesssim M_{\mathrm{r}} \lesssim 1.20 M_{\odot}$ in Fig. 8. A comparison with the rotating model shows that the carbon mass fraction is mostly below 0.5 , even though the rotating model does not suffer from thermal pulses for most of the time. While the average carbon mass fraction in the accreted layer is 0.58 in the non-rotating model, it is 0.45 in the layer with $1.0<M_{\mathrm{r}} / M_{\odot}<1.2$ of the rotating model. The mean value of $X_{\mathrm{C}}$ throughout the accreted layer in the rotating model (i.e., $1.0<M_{\mathrm{r}} / M_{\odot}<1.48$ ) is 0.38 .

The carbon and oxygen mass fractions in the accreted envelope of a SN Ia progenitor are important from the point of view of nucleosynthesis and supernova spectroscopy, and may even affect the peak brightness of the supernova explosion (cf. Umeda et al. 1999; Höflich et al. 2000; Domínguez et al. 2001).

\section{Stability of helium shell burning}

Our results show that helium shell burning is more stable with rotation, than in the non-rotating case (Figs. 1, 3 and 4). The stability of a helium shell source is mainly determined by three factors: geometrical thickness, degree of degeneracy and temperature of the shell source. In other words, a helium shell source is more stable if it is thicker, less degenerate and hotter. While a general and quantitative stability criterion for shell sources has been worked out in a separate paper
(Yoon et al. 2004), we discuss the qualitative effects of rotation on the shell source stability of the models presented in Sect. 3 in the following.

Figure 9 shows the evolution of the helium shell sources of sequences NR4 and R4 in the plane of the degeneracy parameter $\eta(:=\psi / k T$, e.g., Clayton 1968) and the relative thickness of the shell source $D / r_{\mathrm{s}}$, where $D$ and $r_{\mathrm{s}}$ are the thickness and the radius of the shell source, respectively. Here, the energy generation rate weighted mean over the shell source is used for the degeneracy parameter. The thickness of the shell source is defined such that the energy generation rate at each boundary is $2 \times 10^{-3}$ times its peak value. Figure 9 illustrates the evolution toward instability for the example of sequences NR4 and R4. In sequence NR4, the mean temperature in the shell source remains nearly constant, around $T \simeq 2.02 \times 10^{8} \mathrm{~K}$ during the stable shell burning phase. Figure 9 shows the border between stability and instability for $T=2 \times 10^{8} \mathrm{~K}$, as obtained by Yoon et al. (2004), as a dotted line. The shell source becomes more degenerate and thinner as the white dwarf mass grows, and finally enters the unstable regime when $M_{\mathrm{WD}} \simeq 1.03 M_{\odot}$, resulting in the onset of thermal pulses as shown in Fig. $4 \mathrm{c}$.

As already discussed in Sect. 5, the shell source in the rotating models extends over a wider mass range due to rotationally induced chemical mixing. I.e., significant amounts of $\alpha$-particles are mixed into the CO core and participate in the ${ }^{12} \mathrm{C}(\alpha, \gamma){ }^{16} \mathrm{O}$ reaction. The presence of the centrifugal force lowers the density, and were it not for the effect of mixing the shell source temperature would decrease with rotation. However, due to the enhanced ${ }^{12} \mathrm{C}(\alpha, \gamma){ }^{16} \mathrm{O}$ reaction, temperature and nuclear energy production are increased, despite the lower density. In summary, rotation changes all three physical properties which affect the stability - thickness, degeneracy and temperature - such that stability becomes more likely. Figure 9 shows that this effect is quite significant. While the evolutionary tracks of sequences NR4 and R4 are close to each other in the shell thickness-degeneracy plane, at a given mass the shell thickness is much larger and the degeneracy much lower in the rotating models. For example, at $M_{\mathrm{WD}}=1.01 M_{\odot}$, the non-rotating model gives $\eta \simeq-2.9$ and $D / r_{\mathrm{s}} \simeq 0.225$ and the corresponding rotating model gives $\eta \simeq-3.0$ and $D / r_{\mathrm{s}} \simeq 0.281$. Furthermore, we have $\eta \simeq-2.8$ and $D / r_{\mathrm{s}} \simeq 0.19$ at $M_{\mathrm{WD}}=1.03 M_{\odot}$, from which point thermal pulses become significant in the non-rotating sequence, while $\eta \simeq-2.9$ and $D / r_{\mathrm{s}} \simeq 0.25$ even when $M_{\mathrm{WD}}$ is as large as $1.14 M_{\odot}$ in the rotating model (Fig. 9).

Additionally, the instability border which is given by the thin lines in Fig. 9 is located lower at a given mass in the rotating case, due to the higher shell source temperature in the rotating models, which leads to a later onset of the instability even for a given shell source thickness and degeneracy. For instance, the shell source temperature in the rotating model when $M_{\mathrm{WD}}=1.01 M_{\odot}$ is about $2.1 \times 10^{8} \mathrm{~K}$, which is higher than in the non-rotating models, where $T_{\text {shell }}$ remains at $\sim 2.0 \times 10^{8} \mathrm{~K}$ during the steady shell burning phase. The last analyzed model in sequence NR4 is already in the unstable regime (i.e., below the dotted line; $D / r_{\mathrm{s}} \simeq 0.19$ ) and thermal pulses become significant right after as expected from the stability criterion. On the other hand, in sequence R4, the shell source temperature 


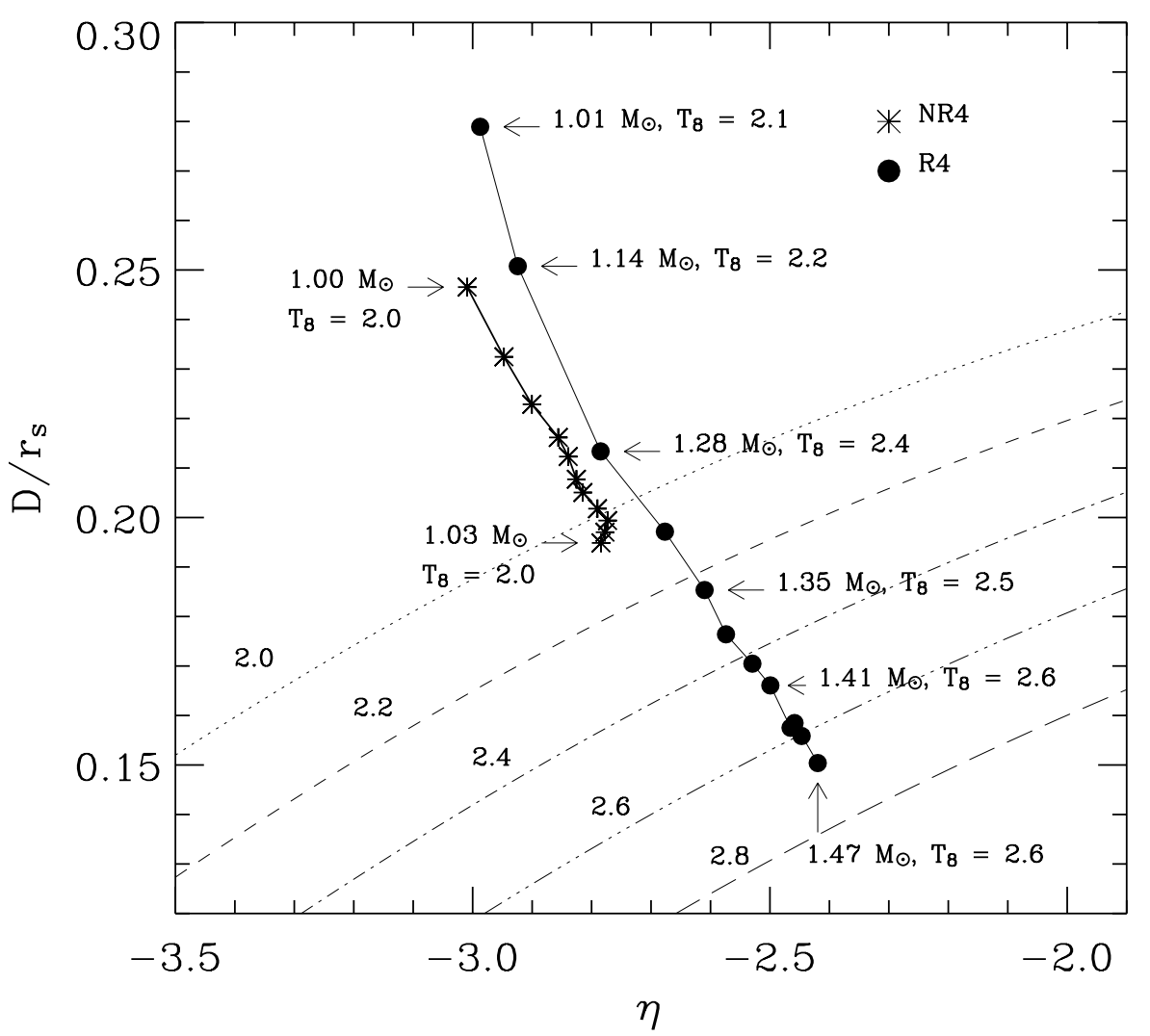

Fig. 9. Evolution of the helium shell source in the sequences NR4 and R4 during the stable shell burning phase, in the plane spanned by the degeneracy parameter $\eta$ and the relative thickness of the shell source $D / r_{\mathrm{s}}$. Here $D$ denotes the thickness of the shell source and $r_{\mathrm{s}}$ its radius. Each asterisk and black dot represents one selected stellar model in sequences NR4 and R4, respectively. The white dwarf mass in solar masses and the temperature of the shell source in units of $10^{8} \mathrm{~K}$ are indicated for various models. The thin lines separate the stable and unstable regimes, for $T /\left(10^{8} \mathrm{~K}\right)=2.0$ (dotted line), 2.2 (short dashed line), 2.4 (dotted-dashed line), 2.6 (three dotted - dashed line) and 2.8 (long dashed line) according to the analytic stability criterion of Yoon et al. (2004). I.e., helium shell burning is predicted to be stable (unstable) above (below) the lines, while thermal pulses become significant from $M_{\mathrm{WD}} \simeq 1.03 M_{\odot}$ in sequence NR4, and weak thermal pulses appear from $M_{\mathrm{WD}} \simeq 1.41 M_{\odot}$ in sequence R4.

increases up to $2.6 \times 10^{8} \mathrm{~K}$ when $M_{\mathrm{WD}}=1.41 M_{\odot}$, which explains why the shell source is stable until $M_{\mathrm{WD}} \simeq 1.41 M_{\odot}$ even though $D / r_{\mathrm{s}}$ at this moment is smaller than in the last analyzed model of NR4. As shown in Fig. 4d (see also Fig. 5), the ensuing thermal pulses which appear when $M_{\mathrm{WD}} \gtrsim 1.41 M_{\odot}$ in sequence $\mathrm{R} 4$ remain very weak until the last model $\left(M_{\mathrm{WD}}=\right.$ $1.48 M_{\odot}$ with $T_{\text {shell }} \simeq 2.6 \times 10^{8} \mathrm{~K}$ ), which is still near the stability border line for $T=2.6 \times 10^{8} \mathrm{~K}$ in Fig. 9 .

In summary, rotation stabilizes the instability of the helium shell burning in our models significantly. The shell sources in realistic white dwarfs, however, may be even more stable than our models predict, for two reasons. Firstly, as outlined in Sect. 2, our method forces us to limit the structural effects of rotation to that obtained at about $60 \%$ of critical rotation. This means that we underestimate the centrifugal force in the layers which contain the shell source. Secondly, our models are one-dimensional, which restricts the pressure response due to any perturbation to the radial direction - which is generally not true in the realistic situation. In a rapidly rotating star, the pressure can also respond to a perturbation horizontally, unless the shell source is located at the poles. We believe that this multi-dimensional effects can stabilize the shell source further.

\section{Concluding discussion}

We have performed numerical simulations of helium-accreting CO white dwarfs with an initial mass of $0.998 M_{\odot}$ and 4 different mass accretion rates $\left(2,3,5\right.$ and $\left.10 \times 10^{-7} M_{\odot} / y r\right)$, considering spin-up by mass accretion. The main feature of the spin-up is the angular momentum transport from the accreted matter into the white dwarf, which creates differential rotation in the white dwarf interior. This induces transport of chemical species from the accreted matter into the CO core, through the shear instability, Eddington-Sweet circulations, and the GSF instability.

It is shown that the helium burning layer becomes spatially more extended than in the non-rotating case, since the accreted $\alpha$-particles are mixed into the carbon enriched layer, participating actively in the ${ }^{12} \mathrm{C}(\alpha, \gamma){ }^{16} \mathrm{O}$ reaction (Sect. 5). The carbonto-oxygen ratio is significantly reduced in the accreted layer. The centrifugal force lowers the density in the shell source and degeneracy is significantly lifted. The total energy generation in the helium shell source increases with rotation, due to the increased contribution from the ${ }^{12} \mathrm{C}(\alpha, \gamma){ }^{16} \mathrm{O}$ reaction, rendering the helium shell source as hot as in the non-rotating cases, despite the decreased density. All these effects contribute to 
stabilizing the helium shell burning significantly (Sect. 6). Thermal pulses, if they occur, are considerably weakened with rotation.

As mentioned previously, and as discussed by Cassisi et al. (1998), Kato \& Hachisu (1999) and Langer et al. (2002), a high mass accumulation efficiency on a white dwarf by hydrogen accretion has been seriously questioned due to the unstable helium shell burning which is found when helium is accreted at a rate which gives steady hydrogen shell burning. Note that the accretion rate of $5 \times 10^{-7} M_{\odot} /$ yr corresponds roughly to the maximum rate for the steady hydrogen burning in a $1.0 M_{\odot}$ white dwarf (Nomoto 1982a). Our results indicate that even if hydrogen is accreted and burned steadily, the subsequent helium shell burning may be unstable, with strong helium shell flashes driving the stellar envelope to the Eddington limit, unless effects of rotation are considered. Kato \& Hachisu (1999) suggested that if the new OPAL opacities are adopted, an optically thick wind can remove mass from the system without the white dwarf filling its Roche lobe. They also suggested that the effective mass accumulation rate can remain high enough for the white dwarf to grow to the Chandrasekhar limit. However, their results are based on a very massive white dwarf model of $1.3 M_{\odot}$. Cassisi et al. (1998), on the other hand, showed that hydrogen-accreting white dwarfs with initial masses of $0.56 M_{\odot}$ and $0.8 M_{\odot}$ fill their Roche lobe once helium flashes occur, leaving doubts about the efficient mass increase of the white dwarf from a relatively low mass by hydrogen accretion.

Our results imply that rotation may help white dwarfs to grow in mass by stabilizing helium shell burning. In the canonical single degenerate scenario, however, a main sequence star or a red giant is supposed to transfer hydrogen-rich matter onto the white dwarf (Li \& van den Heuvel 1997; Hachisu et al. 1999; Langer et al. 2000). We still need to investigate the case of hydrogen accretion, in order to answer the question whether rotation may be a potential solution in explaining the observed $\mathrm{SNe}$ Ia rate from the considered binary systems. Since the stability conditions for hydrogen shell burning are not qualitatively different from that for helium shell burning (Yoon et al. 2004), we expect that the effects of rotation on the hydrogen shell source are likely to give a similar conclusion as for the helium shell burning.

On the other hand, hydrogen accretion with a rate of $10^{-6} M_{\odot} /$ yr is supposed to expand a $1.0 M_{\odot}$ white dwarf into a red giant phase (Nomoto 1982a; Nomoto \& Kondo 1991; Cassisi et al. 1998). In a close binary system, the hydrogen envelope expanded to the red giant phase will fill the Roche lobe, causing significant mass loss or the merging of both stars, unless the mass accretion rate is reduced by an optically thick stellar wind, as suggested by Hachisu et al. (1996, 1999). However, steady helium accretion with such a high accretion rate can be realized, for example, in a binary system which consists of a white dwarf and a helium giant star ("helium Algols", Iben \& Tutukov 1994), in which the mass transfer rate can amount to $10^{-6}-10^{-5} M_{\odot} / \mathrm{yr}$, as shown by Yoon \& Langer (2003). Our results indicate clearly that the mass accumulation efficiency can be significantly enhanced by rotation for helium giant star + white dwarf binary systems. Although the calculated production rate of SNe Ia via this route is currently rather uncertain (Iben \& Tutukov 1994; Branch et al. 1998), consideration of rotation may increase it strongly.

In the present study, we did not consider stellar winds from white dwarfs, on which rotation might cause significant effects. When the stellar surface approaches the critical velocity, the mass loss due to radiation-driven winds could increase dramatically (Friend \& Abbott 1986; Langer 1997). Since stellar winds may also carry a large amount of angular momentum (Langer 1998), this would involve a complicated history of angular momentum gain or loss in accreting white dwarfs: accretion will spin up white dwarfs, while stellar winds will brake them. It is also possible that the physical conditions in the shell source in a rapidly rotating white dwarf vary with latitude, which may lead to interesting consequences such as non-spherical stellar winds (cf. Owocki \& Gayely 1997; Maeder 1999). We will investigate these effects systematically in the near future.

Finally, we note that an increase in the uncertain efficiency of rotationally induced chemical mixing results in more stable shell burning (Figs. 1 and 3), than when the calibration by Heger et al. (2000) and Heger \& Langer (2000) is adopted. Their calibration gives a chemical mixing efficiency similar to that in Pinsonneault et al. (1989) who made a calibration to reproduce the solar surface ${ }^{7} \mathrm{Li}$ abundance, as well as to the theoretical estimate by Chaboyer \& Zahn (1992). The physical conditions in the helium shell source in white dwarfs do not differ qualitatively from those in main sequence stars. However, for accreting white dwarfs, another mixing mechanism which was not considered in the present study may be important: magnetic instability induced by differential rotation in radiative layers (Spruit 2002). Heger et al. (2003) and Maeder \& Meynet (2003) show that the turbulent diffusion can become stronger by several orders of magnitude due to the magnetic instability, than in the case where only rotational effects are considered. The differential-rotation-induced magnetic instability can be even more important in accreting white dwarfs, in which a strong shear motion is retained due to the continuous angular momentum transport from the accreted matter, as shown in Sect. 4.2. The efficiency of the chemical mixing may increase accordingly, which may stabilize the shell source even further than in the present study. This possibility is currently under investigation.

Acknowledgements. We would like to thank the anonymous referee for useful comments and careful reading of the manuscript, which led to significant improvement of the text. We are grateful to Peter Höflich for enlightening discussions. This research has been supported in part by the Netherlands Organization for Scientific Research (NWO).

\section{References}

Branch, D., Livio, M., Yungelson, L. R., Boffi, F. R., \& Baron, E. 1995, PASP, 107, 1019

Branch, D. 1998, ARA\&A, 36, 17

Cassisi, S., Iben, I., \& Tornambé, A. 1998, ApJ, 496, 376

Caughlan, G. R., Fowler, W. A., Marris, M. J., \& Zimmerman, B. A. 1985, Atomic Dat. Nuc. Dat. Tables, 32, 197

Chaboyer, B., \& Zahn, J.-P. 1992, A\&A, 253, 173

Chandrasekhar, S. 1970, Phys. Rev. Lett., 24, 611

Clayton, D. D. 1968, Principles of Stellar Evolution and Nucleosynthesis (New York: MacGraw-Hill) 
Domínguez, I., Höflich, P., \& Straniero, O. 2001, ApJ, 557, 279

Durisen, R. H. 1975, ApJ, 199, 179

Durisen, R. H. 1977, ApJ, 213, 145

Durisen, R. H., \& Imamura, J. N. 1981, ApJ, 243, 612

Endal, A. S., \& Sofia, S. 1976, ApJ, 210, 184

Fliegner, J. 1993, Diplomarbeit, University of Göttingen

Friedman, J. L., \& Schutz, B. 1978, ApJ, 222, 281

Friend, D. S., \& Abbott, D. C. 1986, ApJ, 311, 701

Fujimoto, M. Y. 1988, A\&A, 198, 163

Fujimoto, M. Y., \& Iben, I. 1997, in Advances in Stellar Evolution (Cambridge University Press)

Fujimoto, M. Y., \& Sugimoto, D. 1979, PASJ, 31, 1

Greiner, J. 2000, New Astron., 5, 137

Hachisu, I. 1986, ApJS, 62, 461

Hachisu, I., \& Kato, M. 2001, ApJ, 558, 323

Hachisu, I., Kato, M., \& Nomoto, K. 1996, ApJ, 470, L97

Hachisu, I., Kato, M., \& Nomoto, K. 1999, ApJ, 522, 487

Hamuy, M., Phillips, M. M., \& Suntzeff, N. B., et al. 1996, ApJ, 519, 314

Heber, U., Napiwotzki, R., \& Reid, I. N. 1997, A\&A, 323, 819

Heger, A., \& Langer, N. 2000, ApJ, 544, 1016

Heger, A., Langer, N., \& Woosley, S. E. 2000, ApJ, 528, 368

Heger, A., Woosley, S., Langer, N., \& Spruit, H. C. 2003, in Stellar Rotation, ed. A. Maeder, \& P. Eenens (San Francisco: ASP), Proc. IAU-Symp., 215, in press

Hillebrandt, W., \& Niemeyer, J. C. 2000, ARA\&A, 38, 191

Höflich, P., Khokhlov, A., Wheeler, J. C., et al. 1996, ApJ, 472, 81

Höflich, P., Nomoto, K., Umeda, H., \& Wheeler, J. C. 2000, ApJ, 528, 590

Howell, D. A., Höflich, P., Wang, L., \& Wheeler, J. C. 2001, ApJ, 556, 302

Hujeirat, A. 1995, A\&A, 295, 268

Iben, I. Jr., \& Renzini, A. 1983, ARA\&A, 27, 271

Iben, I. Jr., \& Tutukov, A. V. 1991, ApJ, 370, 615

Iben, I. Jr., \& Tutukov, A. V. 1994, ApJ, 431, 264

Iglesias, C. A., \& Rogers, F. J. 1996, ApJ, 464, 943

Imamura, J. N., Toman, J., Durisen, R. H., Pickett, B., \& Yang, S. 1995, ApJ, 444, 363

Kahabka, P., \& van den Heuvel, E. P. J. 1997, ARA\&A, 35, 69

Karl, C. A., Napiwotzki, R., \& Nelemans, G., et al. 2003, A\&A, 410, 663

Kasen, D., Nugent, P., \& Wang, L., et al. 2003, ApJ, 593, 788

Kato, M., \& Hachisu, I. 1999, ApJ, 513, L41

Kawaler, S. D. 2003, in Stellar Rotation, ed. A. Maeder, \& P. Eenens, Proc. IAU Symp., 215

Kippenhahn, R., \& Möllenhoff, C. 1974, Ap\&SS, 31, 117

Kippenhahn, R., \& Thomas, H.-C. 1970, in Stellar Rotation, ed. A. Slettebak (Dortrecht: Reidel), IAU Coll., 4

Kippenhahn, R., \& Thomas, H.-C. 1978, A\&A, 63, 265

Koester, D., Dreizler, S., Weidemann, V., \& Allard, N. F. 1998, A\&A, 338, 617

Koester, D., Napiwotzki, R., \& Christlieb, N., et al. 2001, A\&A, 378, 556

Langer, N. 1997, in Luminous Blue Variables: Massive Stars in Transition, ed. A. Nota, \& H. J. G. L. M. Lamers, ASP Conf. Ser., 120,83

Langer, N. 1998, A\&A, 329, 551

Langer, N., Heger A., Wellstein, S., \& Herwig, F. 1999, A\&A, 346, L37

Langer, N., Deutschmann, A., Wellstein, S., \& Höflich, P. 2000, A\&A, 362, 1046

Langer, N., Yoon, S.-C., Wellstein, S., \& Scheithauer, S. 2002, in The Physics of Cataclysmic Variables and Related Objets, ASP Conf. Proc., 261
Leibundgut, B. 2001, ARA\&A, 39, 67

Li, X.-D., \& van den Heuvel, E. P. J. 1997, A\&A, 322, L9

Limongi, M., \& Tornambé, A. 1991, ApJ, 371, 317

Lindblom, L. 1999, Phys. Rev. D, 60, 4007

Livio, M. 2001, in Cosmic evolution, ed. E. Vangioni, R. Ferlet, \& M. Lemoine (New Jersey: World Scientific)

Livio, M., \& Pringle, J. E. 1998, ApJ, 505, 339

Livio, M., \& Truran, J. 1987, ApJ, 318, 316

Livne, E., \& Arnett, D. 1995, ApJ, 452, 62

MacDonald, J. 1983, ApJ, 273, 289

Maeder, A. 1999, A\&A, 347, 185

Maeder, A. 2003, A\&A, 399, 263

Maeder, A, \& Meynet, G. 2003, A\&A, 411, 543

Maxted, P. F. L., March, T. R., \& North, R. C. 2000, MNRAS, 317, L41

Meynet, G., \& Maeder, A. 1997, A\&A, 321, 465

Nomoto, K. 1982a, ApJ, 253, 798

Nomoto, K. 1982b, ApJ, 257, 780

Nomoto, K., Iwamoto, K., \& Kishimoto, N. 1997, Science, 276, 1378

Nomoto, K., \& Kondo, Y. 1991, ApJ, 367, L19

Nugent, P., Baron, E., Branch, D., Fisher, A., \& Hauschildt, P. H. 1997, ApJ, 485, 812

Ostriker, J. P., \& Bodenheimer, P. 1968, ApJ, 151, 1089

Ostriker, J. P., \& Mark, J. W.-K. 1968, ApJ, 151, 1075

Owocki, S. P., \& Gayley, K. G. 1997, in Luminous Blue Variables: Massive stars in Transition, ed. A. Nota, \& H. J. G. L. M. Lamers, ASP Conf. Ser., 120

Paczyński, B. 1991, ApJ, 370, 597

Perlmutter, S., Aldering, G., Goldhaber, G., et al. 1999, ApJ, 517, 565

Pinsonneault, M. H., Kawaler, S. D., Sofia, S., \& Demarque, P. 1989, ApJ, 338, 424

Pinto, P. A., Eastman, R. G., \& Rogers, T. 2001, ApJ, 551, 231

Popham, R., \& Narayan, R. 1991, ApJ, 370, 604

Riess, A. G., Filippenko, A. V., Liu, M. C., et al. 2000, ApJ, 536, 62

Ritter, H. 1985, A\&A, 148, 207

Saio, H., \& Nomoto, K. 1985, A\&A, 150, L21

Saio, H., \& Nomoto, K. 1998, ApJ, 500, 388

Sion, E. M. 1999, PASP, 111, 532

Sparks, W. M., \& Kutter, G. S. 1987, ApJ, 321, 394

Schwarzshild, M., \& Härm, R. 1965, ApJ, 142, 855

Starrfield, 2003, in Stellar Rotation, ed. A. Maeder, \& P. Eenens, Proc. IAU Symp., 215

Spruit, H. C. 2002, A\&A, 381, 923

Thoroughgood, T. D., Dhillon, V. S., Littlefair, S. P., Marsh, T. R., \& Smith, D. A. 2001, MNRAS, 327, 1323

Tout, C. A., Regös, E., Wickramasinghe, D., Hurley, J., \& Pols, O. R. 2001, in Evolution of Binary and Multiple Star Systems, ed. Ph. Podsialdlowski et al., ASP Conf. Ser., 229

Umeda, H., Nomoto, K., Kobayashi, C., Hachisu, I., \& Kato, M. 1999, ApJ, 522, L43

Wang, L., Baade, D., Höflich, P., et al. 2003, ApJ, 591, 1110

Weaver, T. A., \& Woosley, S. E. 1993, Phys. Rep., 227, 65

Weigert, A. 1966, Z. Astrophys., 64, 395

Wheeler, C. J. 1996, in Evolutionary Processes in Binary Stars (Dordrecht: Kluwer)

Woosley, S. E., \& Weaver, T. A. 1986, ARA\&A, 24, 205

Woosley, S. E., \& Weaver, T. A. 1994, ApJ, 423, 371

Yoon, S.-C., \& Langer, N. 2002, in The Physics of Cataclysmic Variables and Related Objets, ed. B. T. Gaensicke et al., ASP Conf. Proc., 261

Yoon, S.-C., \& Langer, N. 2003, A\&A, 412, L53

Yoon, S.-C., \& Langer, N. 2004a, A\&A, 419, 623

Yoon, S.-C., \& Langer, N. 2004b, A\&A, 419, 645

Yoon, S.-C., Langer, N., \& van der Sluys, M. 2004, A\&A, 425, 207

Zahn, J.-P. 1975, Mem. Soc. Roy. Liège, 8, 31

Zahn, J.-P. 1992, A\&A, 265, 115 